\title{
Mechanically driven millimeter source of nanosecond X-ray pulses
}

\author{
C.G. Camara · J.V. Escobar · J.R. Hird · S.J. Putterman
}

Received: 8 November 2009 / Revised version: 23 April 2010 / Published online: 14 May 2010

(C) The Author(s) 2010. This article is published with open access at Springerlink.com

\begin{abstract}
The emission of nanosecond pulses of $\approx 20 \mathrm{keV}$ photons having a total energy of GeVs which are generated by peeling millimeter wide strips of pressure sensitive adhesive (PSA) tape in a partial pressure of air $\left(\approx 10^{-3}\right.$ Torr $)$ is demonstrated. The X-ray spectrum is similar to that obtained by peeling much wider bands of PSA, implying that the characteristic length for the sequence of processes that govern this phenomenon is less than $1 \mathrm{~mm}$. These experiments demonstrate that MEMS-type X-ray generators are technologically feasible.
\end{abstract}

\section{Introduction}

Small, independently addressable arrays of X-ray sources would open up new opportunities in the field of computed tomography [1]. Attempts to miniaturize X-ray generators have included the use of carbon nanotubes as electron sources [2]. Such sources (and those used over the course of more than 100 years of X-ray imaging) have, however, relied upon the availability of a high-voltage supply to accelerate electrons to sufficient energies for X-ray emission-a factor that has tended to reduce the portability of such instruments. Recently, it was demonstrated that peeling off-theshelf $3 \mathrm{M}$ Scotch tape in vacuo generates a flux of X-rays that have sufficient spatial coherence and intensity to resolve the skeletal structure of a human digit [3] without the need of high-voltage circuitry. The X-ray emission was interpreted in terms of a two step process. First, the well known but still debated phenomenon of tribo-electricification leads to

C.G. Camara $(\bowtie)$ · J.V. Escobar · J.R. Hird · S.J. Putterman Department of Physics, University of California, Los Angeles, CA 90095, USA

e-mail: camara@physics.ucla.edu a potential difference between the freshly exposed surfaces of the peeling tape. Then there occurs an electric discharge which accelerates electrons to energies where they emit Xrays upon collisions with atoms. To explain the nanosecond duration of the X-ray pulses a specific discharge process was invoked: one initiated by the mutually amplified counter flow of electrons and ions. In order for the X-ray pulses to have nanosecond duration it was argued that the pulses had to originate from a region smaller than a few hundred microns. As a test of this theoretical prediction we have measured X-ray emission from peeling tape that is less than $1.5 \mathrm{~mm}$ wide, which is over a factor of 10 more narrow than previously studied. Consistent with the above theory we find that the reduction of the width of the PSA tape has no effect on the spectral density or temporal characteristics of the emitted X-rays.

\section{Experiment and results}

Figure 1 shows a schematic diagram depicting the size and geometry of the tape peeling-apparatus together with the positioning of the solid state solid-state [Amptek XR-100T CdTe single element and 3-stack and liquid scintillator (LS) [Bicron 501A] X-ray detectors. The solid-state detector was placed $40 \mathrm{~cm}$ from the peeling vertex to maximize the probability of single photon detection, while minimizing pileup. This detector has an active area of $25 \mathrm{~mm}^{2}$, covering $\approx 0.001 \%$ of the $4 \pi$ solid angle. A photomultiplier tube [PMT-Hamamatsu H5783-03] was used to monitor the visible band of triboluminescence (TL) [4] and an exposed $8 \mathrm{~mm}$ length of the central conductive core of a $50 \mathrm{Ohm}$ BNC cable was placed in the vicinity of the peel-line to function as a radio frequency (rf) antenna [5]. 
Fig. 1 (a) Diagram of the peeling $\mathrm{mm}$ tape and the positioning of the photomultiplier tube and various $\mathrm{X}$-ray detectors. The distances $\mathrm{A}, \mathrm{B}$ and $\mathrm{C}$ were $15 \mathrm{~cm}, 40 \mathrm{~cm}$ and $30 \mathrm{~cm}$ respectively. (b) Flash lamp illuminated microscope image of a $1.5 \mathrm{~mm}$ wide strip of tape as it is being peeled at $3 \mathrm{~cm} \mathrm{~s}^{-1}$. The tape has a total thickness of $50 \mu \mathrm{m}$ of which about $10 \mu \mathrm{m}$ is the adhesive. In this picture, the width of the tap appears out of focus to the right of the edge. (c) Comparison of ns X-ray generator to the size of a fingertip
Fig. 2 Signals from the rf antenna (red) the liquid scintillator (black) and the PMT recording the visible TL (blue). The positions of the different signals on the abscissa are corrected for the transit times of the different PMTs and for cable lengths. The cable delay time together with the transit time for the $12.7 \mathrm{~cm}\left(5^{\prime \prime}\right)$ PMT ( $\left.~ 50 \mathrm{~ns}\right)$ was subtracted from the LS events in order to provide a common zero of time. The inset shows a histogram of the width of Gaussian best fits to X-ray flash events as recorded in the LS. The smooth line is a plot for cosmic ray events of similar energy

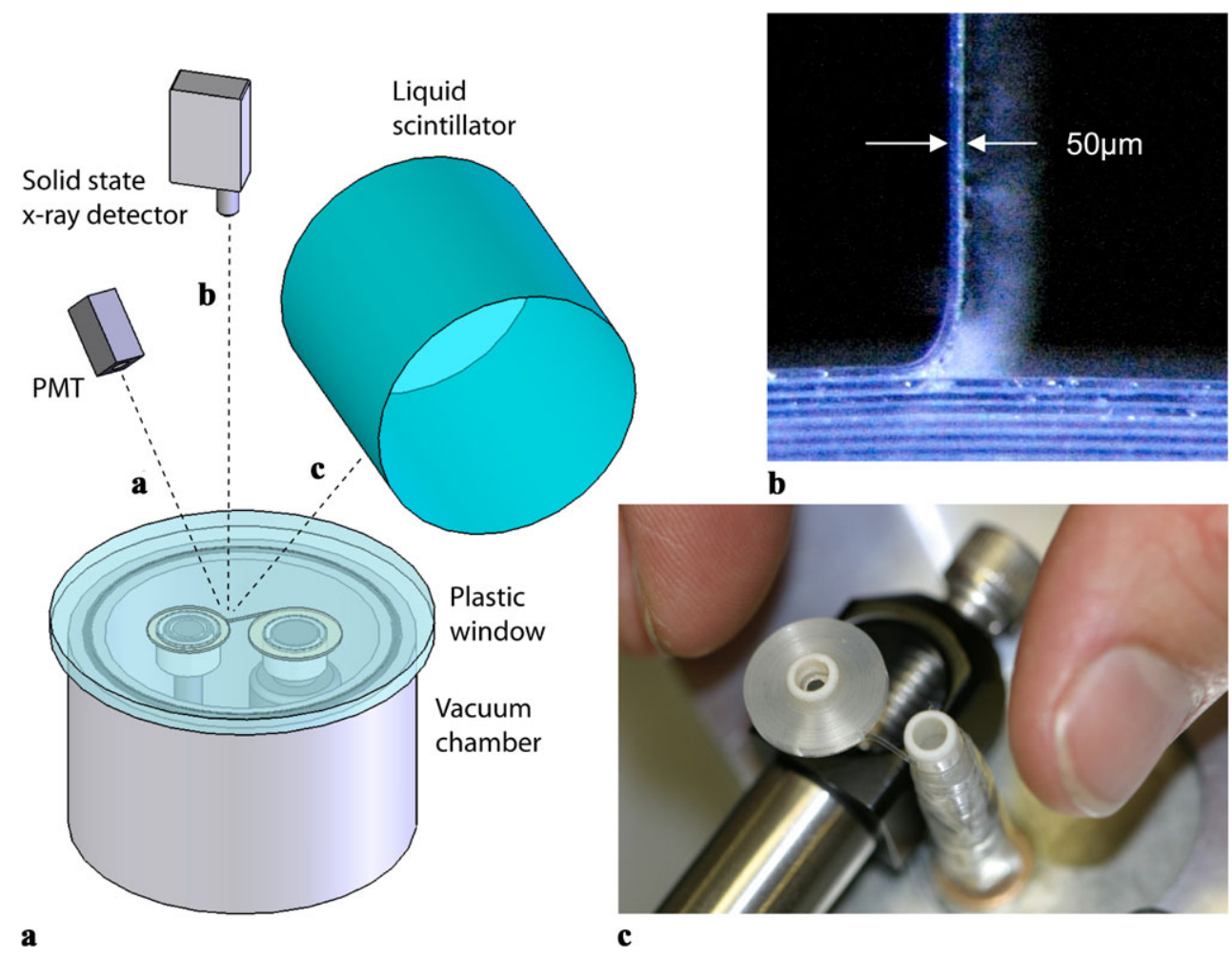

$\mathbf{a}$

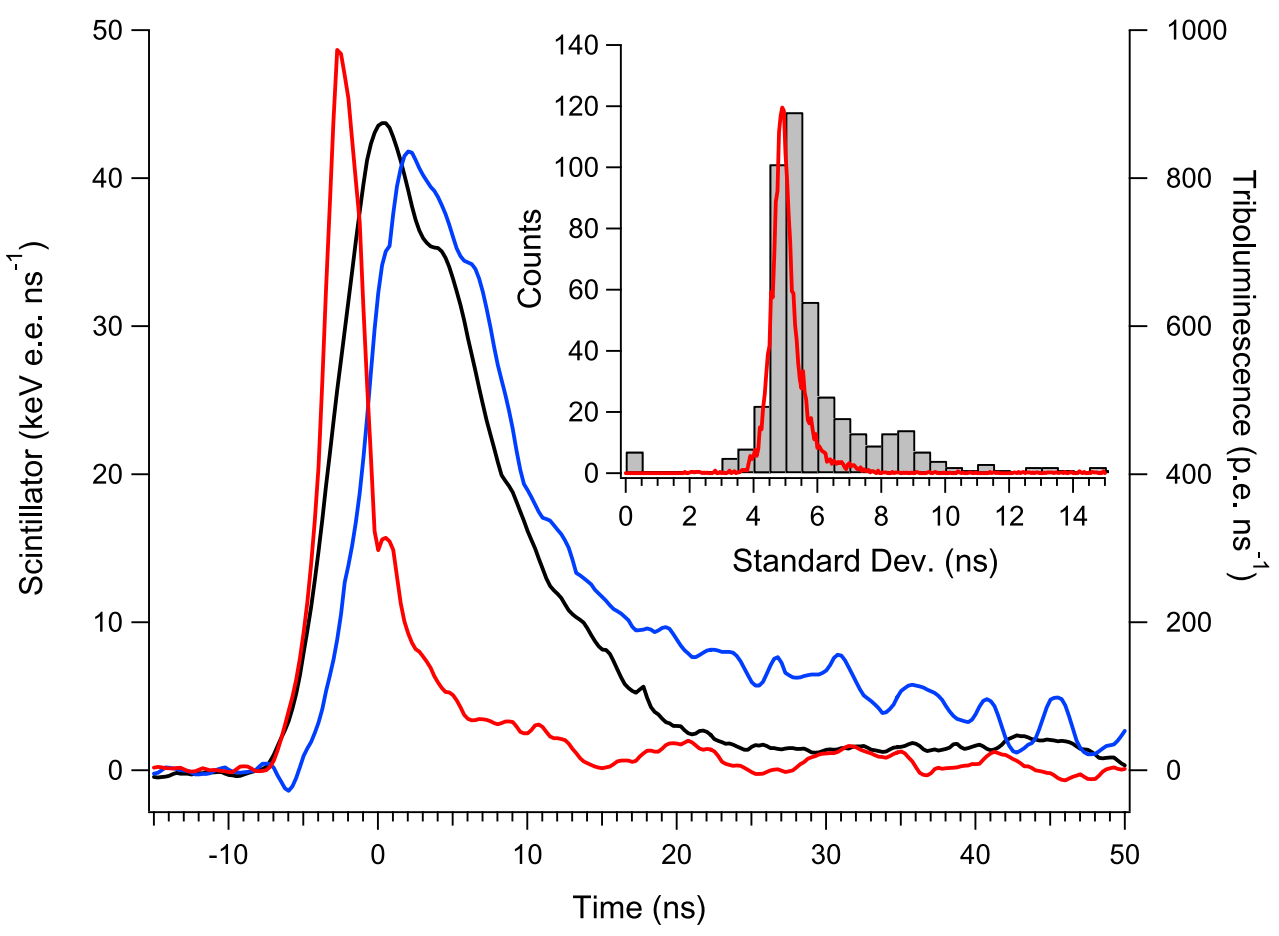

The tape was peeled at $3 \mathrm{~cm} \mathrm{~s}^{-1}$ by a DC motor connected to reducing gears. This set-up was placed inside a vacuum chamber and kept at $10^{-3}$ Torr of air for all the measurements presented here. A $12 \mathrm{~mm}$ thick polycarbonate window on the vacuum chamber allowed X-rays with energies greater than $\approx 10 \mathrm{keV}$ to reach the detectors. Nanosec- ond resolution of the flash width is obtained by recording liquid scintillator flashes with a $12.7 \mathrm{~cm}\left(5^{\prime \prime}\right)$ PMT (not shown in Fig. 1).

Figure 2 shows a comparison of a single shot rf signal with an X-ray pulse from peeling tape, and a visible triboluminescence event recorded on the PMT. The inset to Fig. 2 is 
Fig. 3 X-ray spectra from peeling a $1.5 \mathrm{~mm}$ strip of tape at $10^{-3}$ Torr. The peel speed was between 3 and $3.6 \mathrm{~cm} \mathrm{~s}^{-1}$. The pulse spectrum was acquired with an Amptek CdTe 100XR 3-stack X-ray detector placed $40 \mathrm{~cm}$ from the peeling point looking through a $12 \mathrm{~mm}$ polycarbonate window. The inset shows the single photon $\mathrm{X}$-ray spectra for pulses (black), the spectrum for all the collected $\mathrm{X}$-rays from peeling a single roll of $1.5 \mathrm{~mm} \times 25 \mathrm{~m}$ tape (red) and the spectrum of all the $\mathrm{X}$-rays from peeling a single roll of $1.9 \mathrm{~cm} \times 25 \mathrm{~m}$ tape (blue) divided by 3 for display

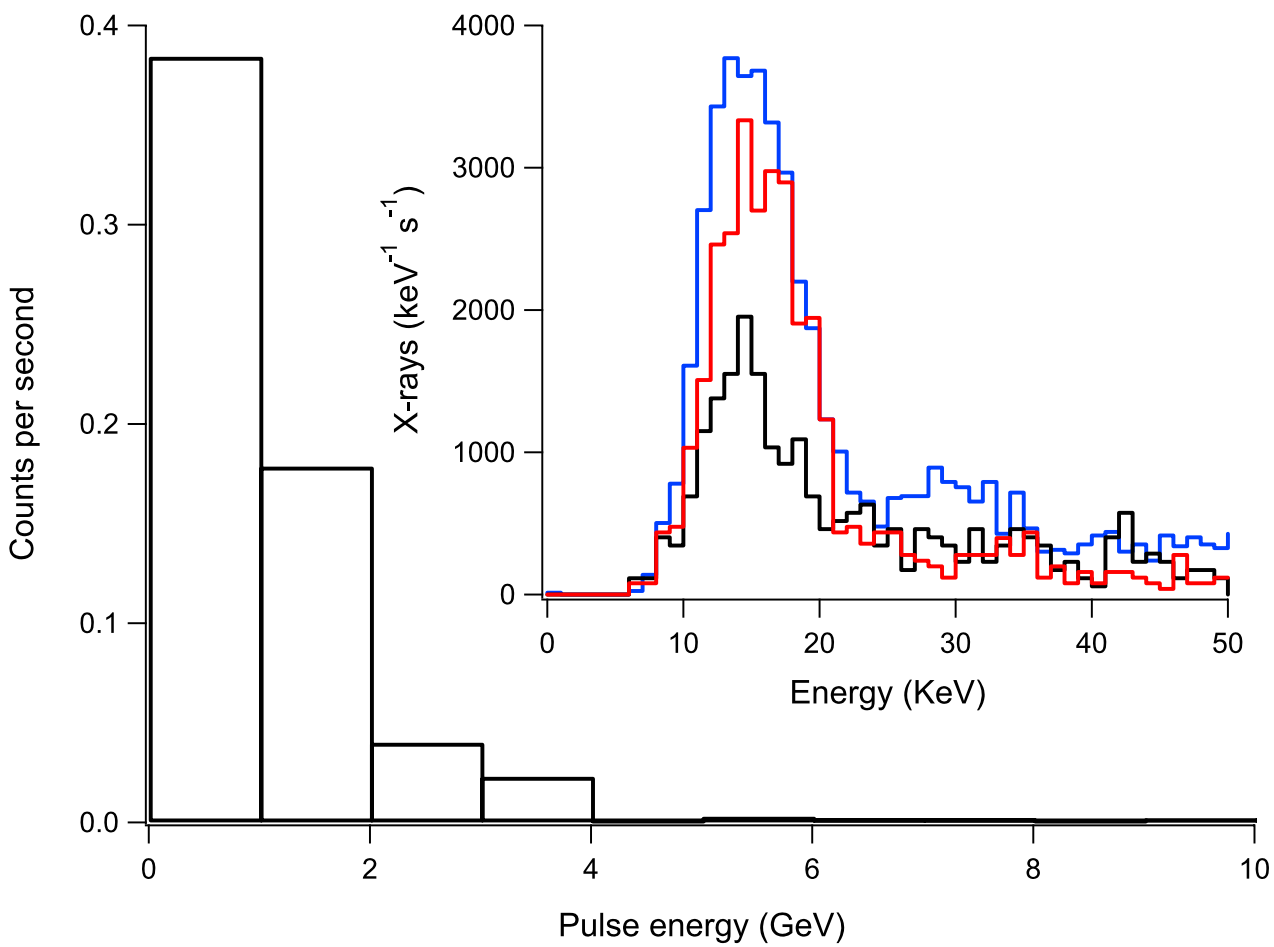

a histogram of the temporal width of Gaussian best fits to Xray events recorded by the LS that are due to peeling $1.5 \mathrm{~mm}$ wide PSA tape. The $\sim 5$ ns best fit width of the majority of these events represents the instrument limit. This was determined by comparison with best fits to cosmic gamma- ray events with approximately the same energy.

Data for the spectra shown in Fig. 3 were acquired by triggering on events measuring over $0.5 \mathrm{~V}$ as detected by the rf antenna. For each trigger, a $10 \mu$ s interval was recorded at 1 point per ns. The complete peeling of a roll of tape took about $700 \mathrm{~s}$ and in a typical run generated $4100 \mathrm{rf}$ triggers. The solid state solid-state detector recorded 532 events with energies above $5 \mathrm{keV}$ that occurred within a $1000 \mathrm{~ns}$ coincidence interval. Of these events, 442 were also coincident with $>10 \mathrm{keV}$ e.e. (electron equivalent energy, [3]) peak in the scintillator within $100 \mathrm{~ns}$ of the rf signal. This conservative time window was chosen to accommodate the different cable delays and detector response times. For these threeway coincidences, the pulse energy was calculated from the solid-state detector corrected for solid angle. The spectrum of photon energies which results from events that are threeway coincident is the black curve in the inset to Fig. 3. The total X-ray photon spectrum for all solid-state detector events is acquired in $10 \mathrm{~s}$ intervals independent of the rf trigger and is plotted on the red curve in the inset. The background for the liquid scintillator was determined from $10 \mu$ s of recorded data outside the $100 \mathrm{~ns}$ coincidence window. During this time, 32 counts with integrated scintillation energy greater than would be produced by a $10 \mathrm{keV}$ electron were observed. These 32 counts per $40 \mathrm{~ms}$ are equivalent to less than one background LS count in the data used to construct Fig. 3.

Figure 4 shows temporally coincident measurements of the emission of visible light as recorded on a PMT [Hamamatsu H5783-03] together with the response of a solid-state $\mathrm{X}$-ray detector that has been placed $10 \mathrm{~cm}$ from the peeling vertex. The PMT is read out through a $1 \mathrm{M} \Omega$ resistor that slows its response time by three orders of magnitude (from ns to $\mu \mathrm{s}$ ). At least three types of correlations can be observed. We interpret the large X-ray pulses that are accompanied by the large TL pulses as being the $4 \pi$ ns Xray pulses resulting from discharges of $10^{10}$ electrons. The $\mathrm{X}$-ray events that correspond to weak TL are interpreted as precursor events in which few X-ray photons are emitted. It is also possible to excite TL without exciting processes which lead to the detection of X-rays.

\section{Discussion}

Bremsstrahlung radiation from electrons with energies over $30 \mathrm{keV}$ is the source of $\mathrm{X}$-rays from peeling tape [3]. The electrons are accelerated by the high electric fields that form as a result of charge separation at the dielectric surfaces of the peeling tape. In analogy to atmospheric lightning, the precise mechanism of charge separation is unknown. Whether the tribo-charging of dielectrics is due to the transfer of ions [6] or electrons [7] is currently debated. A related (and unexplained) observation is that charge can be system- 
Fig. 4 Comparison of the relative timing of visible and $\mathrm{X}$-ray triboluminescence. The upper trace (red) shows the signal recorded by a radio frequency (rf) antenna (arbitrary units). The visible triboluminescence is the middle trace (blue). The lower trace (black) shows X-ray signals recorded by a solid state solid-state X-ray detector

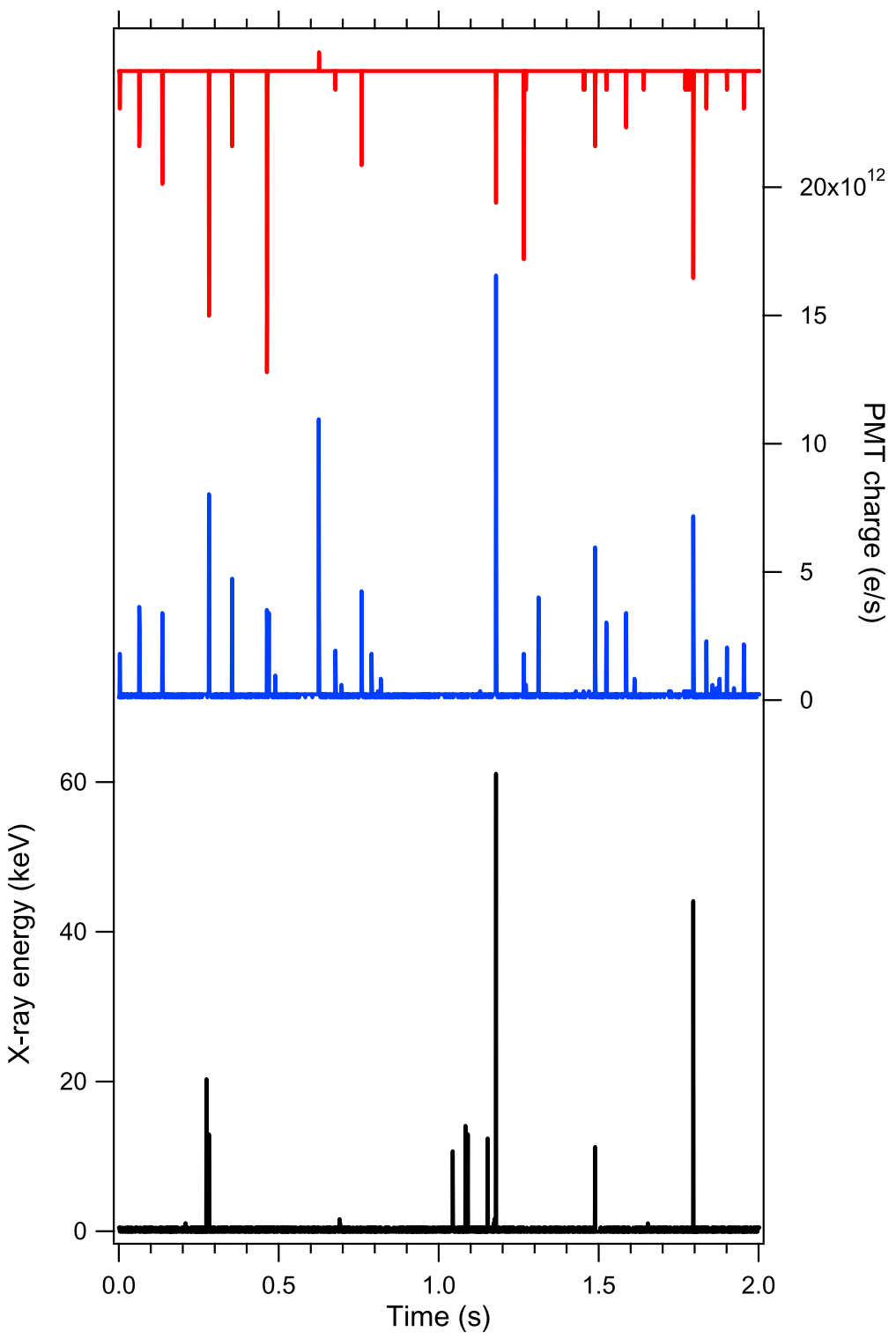

atically transferred between like materials if the radii of curvature of the contacting components is different [8].

A $2 \mathrm{GeV}$ pulse of X-rays contains about $10^{5}$ photons, and a $2 \mathrm{~ns}$ pulse width implies a peak power of $100 \mathrm{~mW}$. In such an event, application of the electron-X-ray conversion efficiency [9] for collisions with carbon leads to an estimate of $10^{10}$ electrons with energy of about $30 \mu \mathrm{J}$. The force required to peel the tape is about $0.15 \mathrm{~N}$ implying an average power input of about $5 \mathrm{~mW}$-one million times smaller than the peak discharge power realized during the ns bursts.

We propose that the build-up of the discharge is due to electrons creating ions at the positively charged surface which then create more electrons at the negatively charged surface, and so on [3]. In this case, the ns pulses are due to a channel that is about $300 \mu \mathrm{m}$ long in a field of $10^{6} \mathrm{~V} \mathrm{~cm}^{-1}$, so that the oppositely charged surfaces each have a charge density of $\sim 7 \times 10^{11}$ fundamental charges per square centimeter.

Another mechanism by which an electric discharge can form involves the generation of secondary electrons via the photoelectric effect [10]. In this case, seed electron(s) create photons as they collide into the positively charged surface. Some of these isotropically emitted photons are intercepted by the negative surface where more electrons are emitted by the photoelectric effect, and so on until the discharge is complete. When photons are the key secondary particle, impact with the negative surface is reduced by the subtended solid angle. In contrast, when positive ions are the key secondary particles in the build-up to a discharge, the negative surface acts as an efficient collector. Although photoelectric effect efficiencies have not been measured for PSA tape, our observation of the same spectral density and pulse energies for 
Fig. 5 Cartoon of a micro mechanical array of independently addressable $\mathrm{X}$-ray sources. 100 sub-mm mechanical actuators could be arranged in this manner. a Cutout view of the array. b Side view of the proposed device to illustrate a fully extended actuator. The entire array would be under vacuum. The top window can be chosen to allow good X-ray transmission. We envision that efficient operation would involve actuation of both longitudinal and transverse motions of the surfaces which are brought into contact

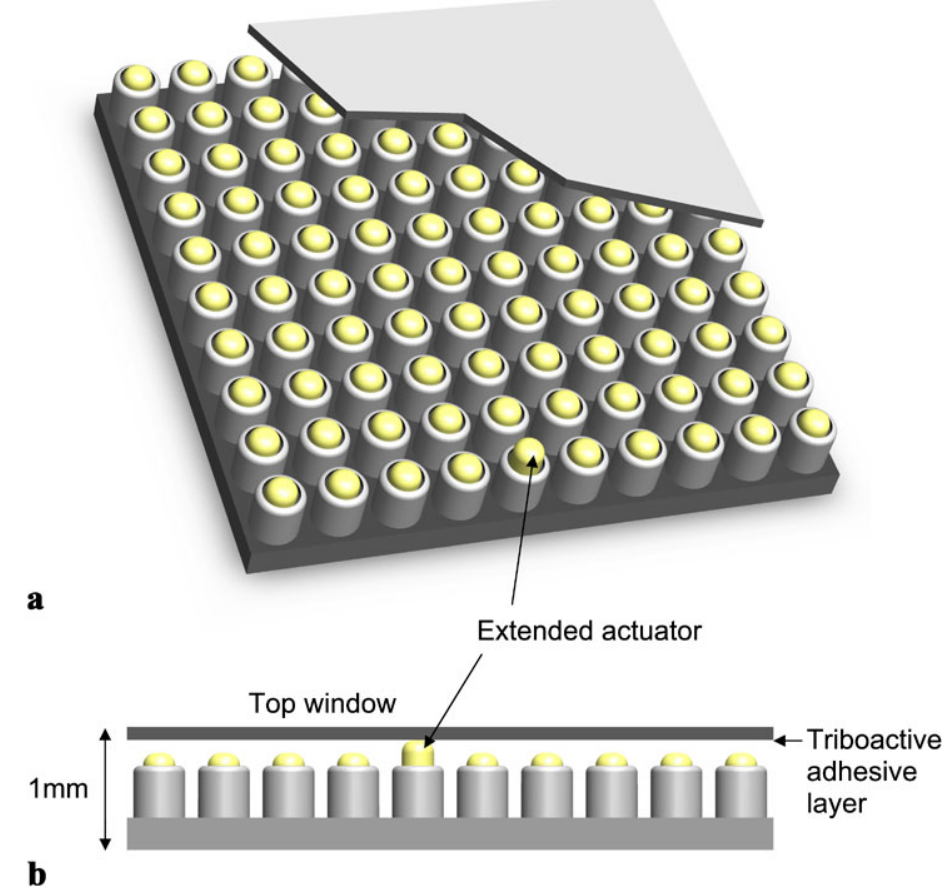

$19 \mathrm{~mm}$ (blue line on inset) and $1.5 \mathrm{~mm}$ wide tape strongly suggest a discharge mechanism mediated by positive ions rather than by the photoelectric effect.

From our estimate of $10^{10}$ electrons per discharge leading to a nanosecond X-ray pulse from surfaces with an unbalanced charge of $7 \times 10^{11} e \mathrm{~cm}^{2}$, it follows that charge must be collected from a square $\mathrm{mm}$ region. This implies that an electrical conductivity parallel to the surface is an important effect which should occur in synchrony with the discharge build-up.

\section{Conclusion}

The emission of X-rays driven by the tribo-charged surfaces of peeling pressure sensitive adhesive tape has been demonstrated for tape that is only $1.5 \mathrm{~mm}$ wide. The build-up of separated charge acts in synchrony with an ion-mediated discharge mechanism and surface conductivity so as to make strong ns X-ray pulses possible. From a technological viewpoint, the X-ray emission is driven mechanically and does not require a high- voltage power supply. This should facilitate the construction of MEMS-type X-ray devices and $\mathrm{X}$-ray sources where each pixel is independently addressable as illustrated in Fig. 5. Impregnation of the surfaces with high $\mathrm{Z}$ materials could lead to order of magnitude improvement in X-ray fluxes. The fundamental limits on the steady state and transient charge densities that can be achieved via contact electrification of dielectrics remains an unknown and its demystification will lead to even greater levels of concentration of mechanical energy into X-ray photons.

Acknowledgements We would like to thank Joseph Dwyer for valuable discussions regarding discharge mechanisms. This research was funded by DARPA.

Open Access This article is distributed under the terms of the Creative Commons Attribution Noncommercial License which permits any noncommercial use, distribution, and reproduction in any medium, provided the original author(s) and source are credited.

\section{References}

1. P.R. Schwoebel, Appl. Phys. Lett. 88, 113902 (2006)

2. H. Choi, J.U. Kim, Mater. Res. Bull. Symp. 963, 0963-Q10-50 (2007)

3. C.G. Camara, J.E. Escobar, J.R. Hird, S.J. Putterman, Nature 455, $1089(2008)$

4. N.E. Harvey, Science 89, 460 (1939)

5. R. Budakian, K. Weninger, R.A. Hiller, S.J. Putterman, Nature (London) 391, 266 (1997)

6. L. McCarty, G.M. Whitesides, Angew. Chem. Int. Ed. 47, 2188 (2008)

7. L. Chongyang, A.J. Bard, Nat. Mater. 7, 505 (2008)

8. E.W.B. Gill, Nature (London) 18, 568 (1948)

9. J.G. Chervenak, A. Liuzzi, Phys. Rev. A 12, 26 (1975)

10. Y. Raizer, Gas Discharge Physics (Springer, Berlin, 1991), p. 132 